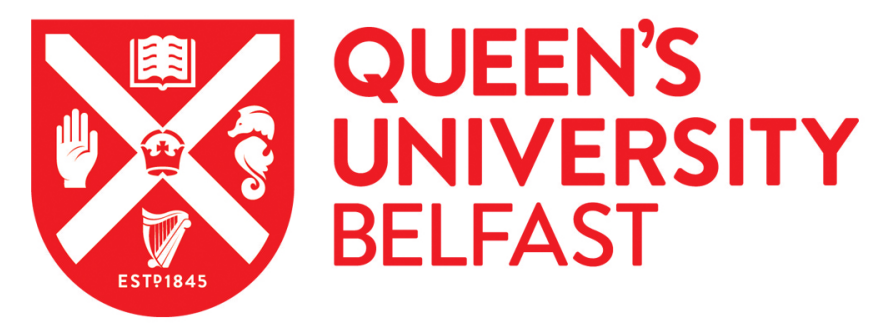

\title{
Higher-Order Terminal Sliding Mode Controller for Fault Accommodation of Lipschitz Second-Order Nonlinear Systems using Fuzzy Neural Network
}

Van, M. (2021). Higher-Order Terminal Sliding Mode Controller for Fault Accommodation of Lipschitz SecondOrder Nonlinear Systems using Fuzzy Neural Network. Applied Soft Computing, 104, [107186].

Published in:

Applied Soft Computing

Document Version:

Peer reviewed version

Queen's University Belfast - Research Portal:

Link to publication record in Queen's University Belfast Research Portal

\author{
Publisher rights \\ Copyright 2021 Elsevier. \\ This manuscript is distributed under a Creative Commons Attribution-NonCommercial-NoDerivs License \\ (https://creativecommons.org/licenses/by-nc-nd/4.0/), which permits distribution and reproduction for non-commercial purposes, provided the \\ author and source are cited.
}

\section{General rights}

Copyright for the publications made accessible via the Queen's University Belfast Research Portal is retained by the author(s) and / or other copyright owners and it is a condition of accessing these publications that users recognise and abide by the legal requirements associated with these rights.

\section{Take down policy}

The Research Portal is Queen's institutional repository that provides access to Queen's research output. Every effort has been made to ensure that content in the Research Portal does not infringe any person's rights, or applicable UK laws. If you discover content in the Research Portal that you believe breaches copyright or violates any law, please contact openaccess@qub.ac.uk. 


\title{
Higher-Order Terminal Sliding Mode Controller for Fault Accommodation of Lipschitz Second-Order Nonlinear Systems using Fuzzy Neural Network
}

\author{
Mien $\operatorname{Van}^{1}$ \\ School of Electronics, Electrical Engineering and Computer Science, Queen's University Belfast, Belfast, \\ United Kingdom
}

\begin{abstract}
In this paper, a higher-order terminal sliding mode control is proposed for fault accommodation of a class of lipschitz second-order nonlinear systems. This approach is designed based on a combining between a novel third-order fast terminal sliding mode surface (TOFTSMS), which is designed to preserve the merits of the PID sliding surface and the fast terminal sliding mode (FTSM) surface, and a continuous control law based on higher-order sliding mode (HOSM) control strategy. However, the proposed TOFTSMC requires an exact dynamics model of the system and the prior knowledge of the bounded value of the uncertainties and faults in the design. In order to exclude the requirements, an adaptive fuzzy neural network is integrated; yielding a novel adaptive fuzzy neural TOFTSMC (AFN-TOFTSMC). The proposed analytical results are then applied to the attitude control of a spacecraft. Simulation results clearly demonstrate the great performance of the proposed algorithm compared to other state-of-the-art methods.

Keywords: Fault tolerant control, Fuzzy neural network, High-order sliding mode control, Nonlinear systems, Robust control.
\end{abstract}

${ }^{1}$ Email: m.van@qub.ac.uk 


\section{Introduction}

In modern machine systems, condition monitoring (CM) and fault diagnosis (FD) have been becoming an important role in designing of the control system to monitor the status and enhance the safety and reliability of the systems [1, 2]. In addition, in 5 some applications in which the system is required to be worked in the hazardous or remote environments, where the humans may face difficult to repair the failed systems, it is highly desired that the system not only can detect the faults but also can accommodate the faults itself and continue working with acceptable performance. Various fault tolerant control (FTC) approaches have been developed to improve system reliability and to guarantee system stability in all situations [3, 4, 5]. Based on the structure of the controller, FTC systems can be broadly divided into two categories: active and passive approaches [4]. In the active approach, FTC system is reconfigured based on the online feedback of the fault information, which is obtained from a fault diagnosis observer [3, 6]. The system performance of the AFTC is, therefore, decreased due to the time delay of the online fault diagnosis scheme and reconfiguration process of the controller. In the passive FTC (PFTC) systems, the FTC is designed as a fixed robust controller without reconfiguration of nominal controller for both normal and fault operations [5, 7, 8]. This approach although requires partial knowledge of a set of the possible system faults, it is simple to implement and provide fast response to the effects of faults. Because the effects of the faults could not be predicted in advance, its effects in the system can be considered as an additional disturbance. To approximate the effects of the disturbance, approximation techniques based on neural networks (NNs) or fuzzy logic systems (FLSs) have been employed in the design of the FTC schemes [9, 10]. Sliding mode control (SMC) [11] is a simple and efficient control method that has been widely used for both linear and nonlinear systems due to its robustness against uncertainties and disturbances $[12,13,14,15]$. The interested robustness property of the SMC has been used for the design of FTC systems [3, 16, 17]. However, the uses of the traditional SMC have four major disadvantages: 1) it provides a large steady state error and slow transient response; 2) it does not converge to the equilibrium point 
the bounded value of the uncertainties in the system.

In the literature, each of the mentioned drawbacks of the conventional SMC has been effectively tackled by a corresponding approach, for example PID-based SMC [18, 19, 20, 21] or its modified version called second-order sliding mode surface (SOSMS)

35 [22, 23] for increasing transient response and decreasing steady-state error, FTSMC for fast finite convergence [24, 25], and HOSM for reducing chattering and increasing the tracking precision [26, 27, 28, 29]. Due to the advantages of the TSMC and the HOSM, second-order terminal sliding mode control (SOTSMC) [30, 31, 32, 33] and full-order sliding mode control (FOSMC) [34] have been developed to obtain both finite time convergence and chattering elimination. Finally, to remove the requirement of the prior knowledge of the bounded value of the uncertainties in the design of the SMCs, adaptive techniques have been developed [35, 23]. However, in many practical applications, it is desired that the system can obtain all three properties such as fast transient response, lower steady-state error, finite time convergence and chatter-

45 ing elimination simultaneously. Another speaking, all drawbacks of the conventional SMC should be eliminated simultaneously using an effective hybrid controller. Unfortunately, there were no approaches in the literature to make this adventure. Therefore, it is needed to further develop a FTC scheme, which can preserve the high robustness property of the conventional SMC while eliminating all drawbacks of it. This is one of 50 the motivations of this paper.

It has been claimed that in order to eliminate all of the aforementioned drawbacks of the conventional SMC, it just needs to design a hybrid control system that can combine the properties of the PID-based SMC, the FTSMC and the HOSMC altogether. From the research point of view, this idea seems potential. However, since the design procedure and properties of the FTSMC, the PID-based SMC and the HOSMC are different, the design of such hybrid system is challenging in our case study. In fact, many issues need to be solved when designing such kind of hybrid controllers, such as how to design a sliding surface that can preserve the full merits of the FTSMC, the PID-based SMC and the HOSM, and how to reconstruct a control input law that can stabilize the system with proper stability proof. From this urgent need, some works have been initialized in our previous approaches to resolve the issues. First, we developed a hybrid 
system that can integrate the properties of two among three controllers. For example, in the first approach [23], we developed a hybrid system that can integrate the properties of the PID-SMC and the HOSMC altogether. In the work [36], we proposed 65 a hybrid controller that can combine the properties of the FTSMC and the HOSMC. Next, we expanded the works to combine the properties of all three controllers, i.e., the PID-based SMC, the FTSMC and the HOSMC. For example, in the work [37] and the work [38], we developed the backstepping nonsingular fast terminal sliding mode control (BNFTSMC) and the PID-nonsingular fast terminal sliding mode control (PID-NFTSMC), respectively. The analyzed results in these studies shown that the two developed controllers have increased the tracking response, obtained finite time convergence and reduced the chattering. However, these two previous approaches have two major disadvantages: (i) the system does not preserve the full merits of the HOSM property in terms of higher tracking precision, and (ii) the chattering of the system is 75 still high and needs to be reduced further. This motivates us to further investigate a new fault tolerant control scheme that can obtain full properties of the HOSMC to increase the performance of the system.

In this paper, in order to preserve the full advantages of the FTSMC, the PIDbased SMC and the HOSM and to compensate the two aforementioned drawbacks of the previous works [37, 38], a novel third-order fast terminal sliding mode control (TOFTSMC) is proposed for robust FTC of a class of second-order nonlinear systems. The proposed TOFTSMC possesses the following two significant properties: 1) the proposed sliding surface preserves the full merits of the PID sliding surface and the FTSM sliding surface, 2) the developed control law is designed based on the HOSM strategy, and thus the proposed controller preserves the full merits of the HOSM: lower chattering and higher tracking precision. Because the proposed TOFTSMC possesses the same properties as the PID-based SMC, the FTSMC and the HOSM, it can overcome all the first three aforementioned disadvantages of the conventional SMC simultaneously [38]. However, the proposed TOFTSMC still requires the exact system 90 dynamics and the prior knowledge of the bounded value of the uncertainties like the conventional SMC. In order to tackle the issue, an adaptive fuzzy neural network (FNN) is also developed in this paper; yielding a novel AFN-TOFTSMC. 
In summary, compared to the existing approaches, the major contributions and novelties of the paper can be summarized as follows:

(i) Compared with the conventional SMC [39, 40, 16, 17], the PID-based SMC [18, 19, 20, 21], the FTSMC [24, 25] and the HOSMC [26, 27, 28, 29], the proposed method, i.e., AFN-TOFTSMC, preserves the merits of all the PID-based SMC, the FTSMC and the HOSMC simultaneously. Thus, the proposed method provides higher tracking performance in terms of fast transient response, lower steady-state error, chattering reduction and finite time convergence.

(ii) Compared with the BNFTSMC [37] and the PID-NFTSMC [38], which do not possess the property of the HOSM controller in terms of enhancing tracking precision, the proposed method is designed based on the HOSM strategy and therefore it can provide higher tracking accuracy. In addition, the use of FNN helps to eliminate the requirement on prior knowledge of the dynamic system and reduce the chattering as well.

The basic properties of the conventional SMC, its advanced controllers and the proposed method, i.e., TOFTSMC, is illustrated in Table 1.

The remainder of this paper is organized as follows. In section 2 , the problem is formulated and some preliminaries are given. The design of the proposed PFTC based on TOFTSMC is presented, in section 3. In section 4, the design of the proposed PFTC based on adaptive fuzzy neural TOFTSMC is described. The simulation results for the attitude control of a spacecraft are given in section 5. The conclusions are given in Section 6. Finally, future recommendations are given in section 7.

\section{Problem formulation and Preliminaries}

\subsection{Problem formulation}

To get more general applications, we consider the following form of second-order nonlinear system [3]:

$$
\begin{aligned}
& \dot{\chi}_{1}=\chi_{2} \\
& \dot{\chi}_{2}=f(\chi)+G(\chi) u_{a}+\varpi
\end{aligned}
$$


Table (1) Properties of the existing advanced sliding mode controllers and the proposed TOFTSMC: TR: Transient Response, FTC: Finite time convergence, SSE: Steady state error

\begin{tabular}{lllll}
\hline \hline Controller & & & & \\
\hline & TR & FTC & SSE & Chattering \\
Conventional SMC [11] & Slow & No & High & Very high \\
FTSMC [24, 25] & Slow & Yes & Fairly high & Very High \\
PID-based SMC[18, 19] & Fast & No & Low & Very high \\
HOSMC[26, 27] & Slow & No & Fairly high & Small \\
Backstepping NFTSMC[37] & Fast & Yes & Low & Small \\
PID-NFTSMC[38] & Fast & Yes & Low & Small \\
Proposed TOFTSMC & Very fast & Yes & Very low & Very Small \\
\hline \hline
\end{tabular}

where $\chi_{1}=\left(x_{1}, \ldots, x_{n}\right)^{T} \in \mathfrak{R}^{n}, \chi_{2}=\left(x_{n+1}, \ldots, x_{2 n}\right)^{T} \in \mathfrak{R}^{n}$ and $\chi=\left(\chi_{1}, \chi_{2}\right)^{T}$ are the vector of system states, $u_{a}=\left(u_{a i}, \ldots, u_{a m}\right) \in \Re^{m}$ with $m \leq n$ denotes the vector of control input, $\varpi=\left(\varpi_{1}, \ldots, \varpi_{n}\right)^{T} \in \Re^{n}$ represents the possible model uncertainties and/or disturbances, $f(\chi) \in \mathfrak{R}^{n}$ and $G(\chi) \in \mathfrak{R}^{n \times m}$ are smooth with $f(0)=0$, and $(\cdot)^{T}$ denotes the transpose of a vector or a matrix.

In this paper, the actuator fault is considered and compensated because it is the most serious failure and often occurs in the system [37]. There are many types of faults that may occur in the actuator, among them gain fault and bias fault are the most often ones. To represent both the gain fault and bias fault generally, the control input in (1) is described as

$$
u_{a}=\Lambda u+\Delta u
$$

where $\Lambda \in \mathfrak{R}^{n}$ and $\Delta u \in \mathfrak{R}^{n}$ represent the gain fault and bias fault, respectively, $u_{a}$ and $u$ are the actual and the desired control value, respectively.

From (1) and (2), the dynamic model (1) can be rewritten as

$$
\begin{aligned}
& \dot{\chi}_{1}=\chi_{2} \\
& \dot{\chi}_{2}=f(\chi)+G(\chi) u+\vartheta(\chi, \Delta u)+\varpi
\end{aligned}
$$


where $\vartheta(\chi, \Delta u)=G(\chi)(\Lambda-I) u+G(\chi) \Delta u \in \mathfrak{R}^{n}$ represents the effects of faults in the system.

To facilitate the design of robust controller to compensate the effects of faults, the effects of the system uncertainties and faults can be summed up to a lumped uncertainty. Hence, the dynamics (3) can be represented as

$$
\begin{aligned}
& \dot{\chi}_{1}=\chi_{2} \\
& \dot{\chi}_{2}=f(\chi)+G(\chi) u+\Xi(\chi, \Delta u, \varpi)
\end{aligned}
$$

where $\Xi(\chi, \Delta u, \varpi)=\vartheta(\chi, \Delta u)+\varpi$ is the lumped uncertainty.

Remark 1. In this paper, FTC system is designed based on the passive approach, which does not require the fault information feedback from a fault diagnosis observer. Therefore, the design of fault diagnosis will not be presented or discussed in this paper. However, the interested readers are recommended to refer to the works [3, 41, 42, 43. 44 36 in the literature for fault diagnosis of nonlinear systems.

Remark 2. The presence of the lumped uncertainty $\Xi(\chi, \Delta u, \varpi)$ reduces the tracking performance significantly. In the severe case, it makes the system unstable and sometime out of control. To handle the effects of the lumped uncertainty, a fuzzy neural network will be employed in this paper to approximate the lumpled uncertainty. The estimated uncertainty is then incorporated into the TOFTSMC to compensate the lumped uncertainty in the system.

\subsection{Preliminaries}

To facilitate in analysis of later sections, we introduce some preliminary results.

Lemma 1. [45]: Consider the nonlinear dynamics system below

$$
\dot{\chi}=f(\chi), f(0)=0, \chi \in \Re^{n}, \chi(0)=\chi_{0}
$$

where $f: D \rightarrow \mathfrak{R}^{n}$ is a continuous on an open neighborhood $D$ of the origin $\chi=0$. Suppose a continuous function $V(\chi): D \rightarrow \Re$ defines on a neighborhood $U \subseteq D$ of the 
origin such that the following conditions hold: $1 . V(\chi)$ is positive definite on $D \subseteq \Re^{n}$; 2. There exists real numbers $a>0$ and $\rho \in(0,1)$, such that $\dot{V}(\chi)+a V^{\rho}(\chi) \leq 0$, then, the system is locally finite-time stable. The settling time, depending on the initial state $\chi(0)=\chi_{0}$, satisfies

$$
T\left(\chi_{0}\right) \leq \frac{V\left(\chi_{0}\right)^{1-\rho}}{a(1-\rho)}
$$

for all $\chi_{0}$ in some open neighborhood of the origin. If $D=\mathfrak{R}^{n}$ and $V(\chi)$ is also unbounded, the system is globally finite-time stable.

Lemma 2. [46]: If the Lyapunov function can be derived as $\dot{V}(\chi)+c_{1} V(\chi)+c_{2} V^{\kappa}(\chi) \leq$ $0, c_{1}, c_{2}>0,0<\kappa<1$, the equilibrium point $\chi=0$ is globally finite-time stable for any given initial condition $\chi(0)=\chi_{0}$, and the convergence time can be calculated as

$$
T \leq \frac{1}{c_{1}(1-\kappa)} \ln \frac{c_{1} V^{1-\kappa}\left(\chi_{0}\right)+c_{2}}{c_{2}}
$$

where $V\left(\chi_{0}\right)$ is the initial value of $V(\chi)$.

\section{PFTC based on third-order fast terminal sliding mode control}

The design procedure of the proposed TOFTSMC consists of two major steps. The first step involves constructing an appropriate sliding surface, which is chosen such that when it converges to zero the desired dynamical characteristics can be achieved. The next step is to design a control law to ensure that sliding motion occurs and that the system states thus converge to zero in finite time.

\subsection{Design of third-order fast terminal sliding mode surface}

For the first step, we propose the use of the following novel third-order fast terminal sliding mode surface (TOFTSMS).

The first-order fast terminal sliding surface is selected as:

$$
s_{i}=\chi_{i}+\int_{0}^{t}\left(h_{1 i} \chi_{i}+h_{2 i}\left|\chi_{i}\right|^{\gamma_{1}} \operatorname{sign}\left(\chi_{i}\right)+h_{3 i}\left|\chi_{i}\right|^{\gamma_{2}} \operatorname{sign}\left(\chi_{i}\right)\right) d t
$$

where $h_{1 i}>0, h_{2 i}>0, h_{3 i}>0,0<\gamma_{1}<1, \gamma_{2}>1(i=1, \ldots, n)$. 
The second-order is designed based on the first-order sliding surface:

$$
\theta_{i}=\dot{s}_{i}+k_{1 i}\left|s_{i}\right|^{\varphi_{1}} \operatorname{sign}\left(s_{i}\right)+k_{2 i}\left|s_{i}\right|^{\varphi_{2}} \operatorname{sign}\left(s_{i}\right)
$$

where $k_{1 i}>0, k_{2 i}>0,0<\varphi_{1}<1, \varphi_{2}>1(i=1, \ldots, n)$.

Finally, the third-order sliding surface is selected as

$$
\sigma_{i}=\dot{\theta}_{i}+m_{1 i}\left|\theta_{i}\right|^{\alpha_{1}} \operatorname{sign}\left(\theta_{i}\right)+m_{2 i}\left|\theta_{i}\right|^{\alpha_{2}} \operatorname{sign}\left(\theta_{i}\right)
$$

where $m_{1 i}>0, m_{2 i}>0,0<\alpha_{1}<1, \alpha_{2}>1(i=1, \ldots, n)$.

Remark 3. Observing from the proposed TOFTSMS (8)-(10), we can see that it has the form of the FTSMC, the PID-based SMC and the FOSMC controllers. Hence, the proposed approach possesses the major advantages of all three controllers simultaneously.

Based on the principle operation of the sliding mode control, the following results can be obtained when the system operates on the sliding mode [12]:

$$
\begin{gathered}
\sigma_{i}=0 \text { and } \dot{\sigma}_{i}=0, i=1, \ldots, n \\
\theta_{i}=0 \text { and } \dot{\theta}_{i}=0, i=1, \ldots, n \\
s_{i}=0 \text { and } \dot{s}_{i}=0, i=1, \ldots, n
\end{gathered}
$$

Combining the results of (10) and (11), we have:

$$
\dot{\theta}_{i}=-m_{1 i}\left|\theta_{i}\right|^{\alpha_{1}} \operatorname{sign}\left(\theta_{i}\right)-m_{2 i}\left|\theta_{i}\right|^{\alpha_{2}} \operatorname{sign}\left(\theta_{i}\right)
$$

Combining the results of (9) and (12), we have:

$$
\dot{s}_{i}=-k_{1 i}\left|s_{i}\right|^{\varphi_{1}} \operatorname{sign}\left(s_{i}\right)-k_{2 i}\left|s_{i}\right|^{\varphi_{2}} \operatorname{sign}\left(s_{i}\right)
$$

Differentiating (8) and subtracting the results to (13), we obtain

$$
\dot{\chi}_{i}=-h_{1 i} \chi_{i}-h_{2 i}\left|\chi_{i}\right|^{\gamma_{1}} \operatorname{sign}\left(\chi_{i}\right)-h_{3 i}\left|\chi_{i}\right|^{\gamma_{2}} \operatorname{sign}\left(\chi_{i}\right)
$$


In the next, we will demonstrate that when the third-order sliding mode motion occurs, i.e., $\sigma_{i}=0$, and the second-order sliding mode motion occurs, i.e., $\theta_{i}=0$, the firstorder sliding mode $s_{i}$ and the system states $\chi_{i}(t)$ will converge to zero in a finite time based on an analysis similar to the approach in [12].

Theorem 1. When the condition in (11) is satisfied, then the origin of the sliding mode dynamics (14) is globally finite-time-stable. The settling time, depending on the initial state $\theta_{i}(0)$, can be determined by $T_{\theta_{i}}\left(T_{\theta_{i}} \leq \frac{\theta_{i}(0) \frac{1-\alpha_{1}}{2}}{\left(1-\alpha_{1}\right) 2 \frac{\alpha_{1}-1}{2} m_{1 i}}\right)$.

Proof. Define the Lyapunov function candidate as follows

$$
V_{1}=\frac{1}{2} \theta_{i}^{2}
$$

From the result of (14) and the derivative of $V_{1}$ with respect to time, we have

$$
\begin{aligned}
\dot{V}_{1} & =\theta_{i} \dot{\theta}_{i}=\theta_{i}\left(-m_{1 i}\left|\theta_{i}\right|^{\alpha_{1}} \operatorname{sign}\left(\theta_{i}\right)-m_{2 i}\left|\theta_{i}\right|^{\alpha_{2}} \operatorname{sign}\left(\theta_{i}\right)\right) \\
& =-m_{1 i}\left|\theta_{i}\right|^{\alpha_{1}+1}-m_{2 i}\left|\theta_{i}\right|^{\alpha_{2}+1} \\
& \leq-2^{\frac{\alpha_{1}+1}{2}} \cdot m_{1 i}\left(\frac{1}{2} \theta_{i}^{2}\right)^{\frac{\alpha_{1}+1}{2}}=-2^{\frac{\alpha_{1}+1}{2}} \cdot m_{1 i} V_{1}^{\frac{\alpha_{1}+1}{2}}
\end{aligned}
$$

And thus,

$$
\dot{V}_{1}+2^{\frac{\alpha_{1}+1}{2}} \cdot m_{1 i} V_{1}^{\frac{\alpha_{1}+1}{2}} \leq 0
$$

By comparing the obtained results in (19) with Lemma 1, we can verify that the origins $\theta_{i}=0, i=1, \ldots, n$ of the sliding mode dynamics (14) are globally stable and convergent to zero in finite time. This completes the proof.

Theorem 2. When the condition (12) is satisfied, then the origin of the sliding mode dynamics (15) is globally finite-time-stable. The settling time, depending on the initial 175 state $s_{i}(0)$, can be determined by $T_{s_{i}}\left(T_{s_{i}} \leq \frac{s_{i}(0)^{1-\varphi_{1}}}{\left(1-\varphi_{1}\right) 2 \frac{\varphi_{1}-1}{2} k_{1 i}}\right)$.

Proof. Define the Lyapunov function candidate as follows

$$
V_{2}=\frac{1}{2} s_{i}^{2}
$$


From the result of (15) and the derivative of $V_{2}$ with respect to time, we have

$$
\begin{aligned}
\dot{V}_{2} & =s_{i} \dot{s}_{i}=s_{i}\left(-k_{1 i}\left|s_{i}\right|^{\varphi_{1}} \operatorname{sign}\left(s_{i}\right)-k_{2 i}\left|s_{i}\right|^{\varphi_{2}} \operatorname{sign}\left(s_{i}\right)\right) \\
& =-k_{1 i}\left|s_{i}\right|^{\varphi_{1}+1}-k_{2 i}\left|s_{i}\right|^{\varphi_{2}+1} \\
& \leq-2^{\frac{\varphi_{1}+1}{2}} \cdot k_{1 i}\left(\frac{1}{2} s^{2}\right)^{\frac{\varphi_{1}+1}{2}}=-2^{\frac{\varphi_{1}+1}{2}} \cdot k_{1 i} V_{2}^{\frac{\varphi_{1}+1}{2}}
\end{aligned}
$$

And thus,

$$
\dot{V}_{2}+2^{\frac{\varphi_{1}+1}{2}} \cdot k_{1 i} V_{2}^{\frac{\varphi_{1}+1}{2}} \leq 0
$$

Comparing the obtained results in (22) with the Lemma 1, we can insist that the origins $s_{i}=0, i=1, \ldots, n$ of the sliding mode dynamics $(15)$ are globally stable and convergent to zero in a finite time. This completes the proof.

Theorem 3. When the condition (13) is satisfied, then the origin of the sliding mode dynamic (16) is globally finite-time-stable. The settling time, depending on the initial state $\chi_{i}(0)$, can be determined by $T_{\chi_{i}} \leq \frac{1}{h_{1 i}\left(1-\gamma_{1}\right)} \ln \left(\frac{2 h_{1 i} \chi_{i}(0)^{\frac{1-\gamma_{1 i}}{2}}+2^{\frac{\gamma_{1 i}+1}{2}} h_{2 i}}{2^{\frac{\gamma_{1 i}+1}{2}} h_{2 i}}\right)$.

Proof. Define the Lyapunov function candidate as follows

$$
V_{3}=\frac{1}{2} \chi_{i}^{2}
$$

From the result of $(16)$ and the derivative of $V_{3}$ with respect to time, we have

$$
\begin{aligned}
\dot{V}_{3} & =\chi_{i} \dot{\chi}_{i} \\
& =\chi_{i}\left(-h_{1 i} \chi_{i}-h_{2 i}\left|\chi_{i}\right|^{\gamma_{1}} \operatorname{sign}\left(\chi_{i}\right)-h_{3 i}\left|\chi_{i}\right|^{\gamma_{2}} \operatorname{sign}\left(\chi_{i}\right)\right) \\
& =-h_{1 i} \chi_{i}^{2}-h_{2 i}\left|\chi_{i}\right|^{\gamma_{1}+1}-h_{3 i}\left|\chi_{i}\right|^{\gamma_{2}+1} \\
& \leq-2 h_{1 i}\left(\frac{1}{2} \chi_{i}^{2}\right)-2^{\frac{\gamma_{1}+1}{2}} \cdot h_{2 i}\left(\frac{1}{2} \chi_{i}^{2}\right)^{\frac{\gamma_{1}+1}{2}}
\end{aligned}
$$

From [24), we obtain

$$
\dot{V}_{3}+2 h_{1 i} V_{3}+2^{\frac{\gamma_{1}+1}{2}} \cdot h_{2 i} V_{3}^{\frac{\gamma_{1}+1}{2}} \leq 0
$$

Therefore, according to Lemma 2, the conclusion holds. This completes the proof. 
Remark 4. Based on the analysis of the FTSMC [24] 25], to guarantee the fast finite time convergence of the sliding mode control, the parameters $\gamma_{1}, \gamma_{2}, \varphi_{1}, \varphi_{2}, \alpha_{1}, \alpha_{2}$ should be chosen as defined right after (8), (9) and (10), respectively. The main reason behind choosing the term with power greater than one and the term with power less than one for these values is to make the convergence of the tracking errors faster and to guarantee finite time convergence. Therefore, when the system is far away from the origin, the term with power greater than one will dominate the fast convergent rate and when the system converges toward the origin, the term with power less than one will dominate the finite time convergence [36]. The constant coefficients $k_{1}, k_{2}, m_{1}, m_{2}, h_{1}, h_{2}, h_{3}$ decides the trade-off between the fast convergence and finite time convergence. These parameters are usually chosen based on trial and error experiments.

Remark 5. The Theorems 1, 2 and 3 are built based on the results of Lemma 1 and Lemma 2 which have been investigated in the literature. However, since the proposed method is formed based on the novel third-order sliding mode sliding surface, i.e., (8), (9) and (10), the novelty of this paper is to integrate the Theorems 1, 2 and 3 altogether so that it can preserve the merits of the FTSMC, the PID-based SMC and the HOSMC simultaneously.

3.2. Design of PFTC based on third-order fast terminal sliding mode control

To design PFTC based on TOFTSMC, the following conditions are assumed.

Assumption 1. The system uncertainties and faults are bounded [3. 37. 38]:

$$
\left\|\Xi(\chi, \Delta u, \varpi)_{i}\right\| \leq l_{\Phi_{i}}, i=1, \ldots, n
$$

where $l_{\Phi_{i}}>0$ is a constant.

Assumption 2. There exists a positive constant $k_{T_{i}}$ such that [37, 38]:

$$
d \Xi_{i}=\left\|\frac{d}{d t} \Xi(\chi, \Delta u, \varpi)_{i}\right\| \leq k_{T_{i}}, i=1, \ldots, n
$$


The above two assumptions are quite general in the literature for designing fault tolerant control system (see references [47, 48, 49]). According to Jiang B [47], this assumption is not really restricted, that is the fault diagnosis and isolation and fault tolerant control are designed for situations where the system is not "exploring". As a consequence, the Lipschitz conditions is indeed satisfied in the bounded region of the state space that is practically considered [47].

as

$$
u_{T O F T S M C}=-G^{+}(\chi)\left(u_{e q}+u_{n}\right)
$$

where,

$$
\begin{aligned}
u_{e q}= & f(\chi)+h_{1} \chi_{2}+h_{2} \gamma_{1}\left|\chi_{1}\right|^{\gamma_{1}-1} \cdot \chi_{2}+h_{3} \gamma_{2}\left|\chi_{1}\right|^{\gamma_{2}-1} \cdot \chi_{2} \\
& +k_{1} \varphi_{1}|s|^{\varphi_{1}-1} \cdot \dot{s}+k_{2} \varphi_{2}|s|^{\varphi_{2}-1} \cdot \dot{s} \\
& +m_{1}|\theta|^{\alpha_{1}} \operatorname{sign}(\theta)+m_{2}|\theta|^{\alpha_{2}} \operatorname{sign}(\theta)
\end{aligned}
$$

and,

$$
\dot{u}_{n}=\left(k_{T}+\eta\right) \operatorname{sign}(\sigma)
$$

where $G^{+}(\chi)=G^{T}(\chi)\left(G(\chi) G^{T}(\chi)\right)^{-1}, u_{n}(0)=0$ and $\eta>0$.

Since the right hand side of (30) contains a discontinuous function, its solution is understood in Filippov sense [50].

Theorem 4. If the nonlinear system described by (4) is controlled by the proposed TOFTSMC law designed as in (28), in which $u_{e q}$ is designed as in (29) and $u_{n}$ is designed as in (30), the stability of the proposed TOFTSMC system can be guaranteed and the third-order sliding surface, i.e., $\sigma_{i}$, will converge to a neighborhood of zero in a finite time. Consequently, the second-order sliding surface, i.e., $\theta_{i}$, the first-order sliding surface, i.e., $s_{i}$, and the system state, i.e., $\chi_{i}$, will converge to zero in a finite time. 
Proof: Differentiating (8) with respect to time and substituting the result into (9), then differentiating (9) with respect to time and substituting the result into [10, we have

$$
\begin{aligned}
\sigma= & \dot{\chi}_{2}+h_{1} \chi_{2}+h_{2} \gamma_{1}\left|\chi_{1}\right|^{\gamma_{1}-1} \cdot \chi_{2}+h_{3} \gamma_{2}\left|\chi_{1}\right|^{\gamma_{2}-1} \cdot \chi_{2} \\
& +k_{1} \varphi_{1}|s|^{\varphi_{1}-1} \cdot \dot{s}+k_{2} \varphi_{2}|s|^{\varphi_{2}-1} \cdot \dot{s} \\
& +m_{1}|\theta|^{\alpha_{1}} \operatorname{sign}(\theta)+m_{2}|\theta|^{\alpha_{2}} \operatorname{sign}(\theta)
\end{aligned}
$$

From (4) and (31), the sliding manifold can be rewritten as

$$
\begin{aligned}
\sigma= & f(\chi)+G(\chi) u+\Xi(\chi, \Delta u, \varpi)+h_{1} \chi_{2}+h_{2} \gamma_{1}\left|\chi_{1}\right|^{\gamma_{1}-1} \cdot \chi_{2} \\
& +h_{3} \gamma_{2}\left|\chi_{1}\right|^{\gamma_{2}-1} \cdot \chi_{2}+k_{1} \varphi_{1}|s|^{\varphi_{1}-1} \cdot \dot{s} \\
& +k_{2} \varphi_{2}|s|^{\varphi_{2}-1} \cdot \dot{s}+m_{1}|\theta|^{\alpha_{1}} \operatorname{sign}(\theta)+m_{2}|\theta|^{\alpha_{2}} \operatorname{sign}(\theta)
\end{aligned}
$$

Substituting the control law $28 \sqrt{30}$ into $[32]$, one yields

$$
\sigma=\Xi(\chi, \Delta u, \varpi)+u_{n}
$$

Substituting (28) and (29) into the derivative of (33), we have

$$
\begin{aligned}
\dot{\sigma} & =d \Xi+\dot{u}_{n} \\
& =d \Xi-\left(k_{T}+\eta\right) \operatorname{sign}(\sigma)
\end{aligned}
$$

Define the following Lyapunov function candidate

$$
V_{4}=\frac{1}{2} \sigma^{T} \sigma
$$

Taking derivative of the above Lyapunov function and combining with the result of (34), we have:

$$
\begin{aligned}
\dot{V}_{4} & =\sigma^{T} \dot{\sigma} \\
& =\sigma^{T}\left(d \Xi-\left(k_{T}+\eta\right) \operatorname{sign}(\sigma)\right) \\
& =d \Xi \sigma-\left(k_{T}+\eta\right)|\sigma| \\
& =\left(d \Xi \sigma-k_{T}|\sigma|\right)-\eta|\sigma| \\
& \leq-\eta|\sigma|
\end{aligned}
$$


Therefore, according to the Lyapunov criteria, the TOFTSMC system guarantees the stability of the third-order sliding surface, i.e., $\sigma$, despite the existing of the parametric uncertainties, external disturbances and faults. Consequently, this result guarantees the stability of the second-order sliding surface, i.e., $\theta$, the first-order sliding surface, i.e., $s$, and the system state, i.e., $\chi$, according to the results in Theorems 1,2 and 3. This completes the proof.

Remark 6. Different from the conventional SMC, where the presence of 'sign' function provide big chattering in the system, the proposed controller $u_{n}$ in (30) used the integration of the sign function, so the chattering in the system can be reduced significantly.

This property takes of the advantage of the higher-order sliding mode control.

\section{PFTC based on adaptive fuzzy neural third-order fast terminal sliding mode control}

In the design of the TOFTSMC, the sliding gains are selected based on the Assumptions 1 and 2. In addition, the design procedure requires an exact model of the dynamic function $f(\chi)$. However, these parameters are difficult to be obtained precisely in advance in real applications for some cases. In this subsection, an adaptive fuzzy-neural network is developed to overcome the limitations.

\subsection{Fuzzy Neural Network}

The FNN is comprised of four layers, i.e., input, membership, rule, and output layers, which contribute to the fuzzy rule base as follows:

$$
\text { If } \chi_{1} \text { is } A_{1}^{i} \text { and } \ldots \text { and } \chi_{n} \text { is } A_{n}^{i} \text { then } y \text { is } B^{i}
$$


where $A_{1}^{i}, A_{2}^{i}, \ldots, A_{n}^{i}$ and $B^{i}$ are fuzzy sets. The output of the FNN's system is defined as

$$
y=\sum_{i=1}^{h} w_{i}\left(\prod_{j=1}^{n} \mu_{A_{j}^{i}}\left(\chi_{j}\right)\right) / \sum_{i=1}^{h}\left(\prod_{j=1}^{n} \mu_{A_{j}^{i}}\left(\chi_{j}\right)\right)=\mathrm{w}^{T} \Psi(\chi)
$$

where $h$ is the number of If-Then rules, and $\mu_{A_{j}^{i}}\left(\chi_{j}\right)$ denotes the membership function value of fuzzy variable $\chi_{j} . \mathrm{w}=\left[w_{1}, w_{2}, . ., w_{h}\right]^{T}$ stands for the adjustable weight madefined as

$$
\psi_{i}(\chi)=\prod_{j=1}^{n} \mu_{A_{j}^{i}}\left(\chi_{j}\right) / \sum_{i=1}^{h}\left(\prod_{j=1}^{n} \mu_{A_{j}^{i}}\left(\chi_{j}\right)\right)
$$

And, $\mu_{A_{j}^{i}}(\chi)$ is chosen as the following form:

$$
\mu_{A_{j}^{i}}(\chi)=\exp \left(\frac{-\left(\chi-c_{i}\right)^{T}\left(\chi-c_{i}\right)}{b_{i}^{2}}\right)
$$

where $b_{i}$ is the width of the Gaussian function and $c_{i}=\left[c_{i 1}, c_{i 2}, \ldots, c_{i n}\right]$ is the center.

\subsection{Design of PFTC based on adaptive fuzzy-neural third-order fast terminal sliding} mode control (AFN-TOFTSMC)

The unknown components in the system (4) can be represented as

$$
M(\chi)=f(\chi)+\Xi(\chi, \Delta u, \varpi)
$$

Denotes $\hat{M}(\chi)$ as the estimation function of $M(\chi)$. The estimation $\hat{M}(\chi)$ can be represented by the output of an integral fuzzy neural network, as follows:

$$
\hat{M}(\chi)=\int_{0}^{t} \hat{\mathrm{w}}^{T}(t) \Psi(\chi) d t
$$

where $\hat{\mathrm{w}}$ denotes the adjustable parameter vector. 
Then, the optimal parameter $\mathrm{w}^{*}$ can be defined as

$$
\mathrm{w}^{*}=\operatorname{argmin}\left\{\sup _{\chi \in U_{\chi}}|M(\chi)-\hat{M}(\chi, \hat{\mathrm{w}})|\right\}
$$

Consequently, $M(\chi)$ is approximated to arbitrary accuracy by an FNN as following Lemma 3.

Lemma 3. [51 52 35]: For any given real continuous function $M(\chi)$ on a compact set $U_{\chi} \in \mathfrak{R}^{n}$ and an arbitrary $\varepsilon$, there exists a fuzzy neural approximator $\hat{M}$ in the form of (42) such that

$$
\sup _{\chi \in U_{\chi}}|M(\chi)-\hat{M}(\chi, \hat{w})|<\varepsilon
$$

The system (4) can be rewritten using a FNN approximator:

$$
\begin{aligned}
& \dot{\chi}_{1}=\chi_{2} \\
& \dot{\chi}_{2}=\int_{0}^{t} \hat{\mathrm{w}}^{T}(t) \Psi(\chi) d t+G(\chi) u+\phi
\end{aligned}
$$

where $\phi=M(\chi)-\int_{0}^{t} \hat{\mathrm{w}}^{T}(t) \Psi(\chi) d t+\varepsilon$ is the lumped uncertainty, and this value and its derivative are assumed to be bounded by :

$$
\phi \leq \bar{\phi} \text { and } d \phi=\frac{d}{d t} \phi \leq K_{\phi}
$$

where $\bar{\phi}$ and $K_{\phi}$ are unknown constants.

An adaptive fuzzy neural TOFTSM control law can be designed as

$$
u_{F N N-T O F T S M C}=-G^{+}(\chi)\left(u_{a e q}+u_{F N N}+u_{a n}\right)
$$

where,

$$
\begin{aligned}
u_{a e q}= & h_{1} \chi_{2}+h_{2} \gamma_{1}\left|\chi_{1}\right|^{\gamma_{1}-1} \cdot \chi_{2}+h_{3} \gamma_{2}\left|\chi_{1}\right|^{\gamma_{2}-1} \cdot \chi_{2} \\
& +k_{1} \varphi_{1}|s|^{\varphi_{1}-1} \cdot \dot{s}+k_{2} \varphi_{2}|s|^{\varphi_{2}-1} \cdot \dot{s} \\
& +m_{1}|\theta|^{\alpha_{1}} \operatorname{sign}(\theta)+m_{2}|\theta|^{\alpha_{2}} \operatorname{sign}(\theta)
\end{aligned}
$$




$$
\dot{u}_{F N N}=\hat{\mathrm{w}}^{T}(t) \Psi(\chi)
$$

$$
\dot{u}_{a n}=\left(\hat{K}_{\phi}+\eta\right) \operatorname{sign}(\sigma)
$$

where, the tuning laws of the FNN's weights and the switching gain $\hat{w}$ and $\hat{K}$ are designed as

$$
\begin{aligned}
& \dot{\hat{\mathrm{w}}}=\frac{1}{\alpha} \sigma \Psi(\chi) \\
& \dot{\hat{K}}_{\phi}=\frac{1}{\lambda}|\sigma|
\end{aligned}
$$

where $\alpha$ and $\lambda$ are the constant adaptive rates. The stability and convergence of the system are stated in Theorem 5.

Theorem 5. Consider the system (4) and the proposed third-order sliding surface in (8)-(10), if the proposed adaptive FNN-TOFTSM control law designed as in 47 52) is used as the control input for the system (4), then the stability of the system can be guaranteed and the third-order sliding surface, i.e., $\sigma_{i}$, will converge to a neighborhood of zero in a finite time. Consequently, the second-order sliding surface, i.e., $\theta_{i}$, the firstorder sliding surface, i.e., $s_{i}$, and the system state, i.e., $\chi_{i}$, will converge to zero in a finite time.

Proof. From (8), 26, and 27, the sliding surface (32) becomes

$$
\sigma=\int_{0}^{t} \tilde{\mathrm{w}}^{T}(t) \Psi(\chi) d t+\phi+u_{a n}
$$
where $\tilde{\mathrm{w}}=\mathrm{w}^{*}-\hat{\mathrm{w}}$ is the FNN's weight approximation error.

Differentiating the sliding surface (53) with respect to time, we have

$$
\dot{\sigma}=\tilde{\mathrm{w}}^{T}(t) \Psi(\chi)+d \phi+\dot{u}_{a n}
$$


Consider the following Lyapunov function

$$
V_{5}=\frac{1}{2} \sigma^{T} \sigma+\frac{1}{2} \alpha \tilde{\mathrm{w}}^{T} \tilde{\mathrm{w}}+\frac{1}{2} \lambda{\tilde{K_{\phi}}}^{T} \tilde{K_{\phi}}
$$

where $\tilde{K_{\phi}}=\hat{K_{\phi}}-K_{\phi}$ is the adaptation gain error.

Differentiating the Lyapunov function (55) and combining the result with (54), we have:

$$
\begin{aligned}
\dot{V}_{5} & =\sigma^{T} \dot{\sigma}+\alpha \tilde{\mathrm{w}}^{T} \dot{\tilde{\mathrm{w}}}+\lambda{\tilde{K_{\phi}}}^{T} \dot{\tilde{K}}_{\phi} \\
& =\sigma^{T}\left(\tilde{\mathrm{w}}^{T}(t) \Psi(\chi)+d \phi+\dot{u}_{a n}\right) \\
& -\alpha \tilde{\mathrm{w}}^{T} \dot{\hat{\mathrm{w}}}+\lambda\left(\hat{K}_{\phi}-K_{\phi}\right) \dot{\hat{K}}_{\phi}
\end{aligned}
$$

Inserting the adaptive law (52)-(51) into [56), we have

$$
\begin{aligned}
\dot{V}_{5} & =\sigma^{T}\left(\tilde{\mathrm{w}}^{T}(t) \Psi(\chi)+d \phi+\dot{u}_{a n}\right) \\
& -\alpha \tilde{\mathrm{w}}^{T} \dot{\hat{\mathrm{w}}}+\lambda\left(\hat{K}_{\phi}-K_{\phi}\right) \dot{\hat{K}}_{\phi} \\
& =\sigma d \phi-K_{\phi}|\sigma|-\eta|\sigma| \\
& \leq-\eta|\sigma|
\end{aligned}
$$

Therefore, based on the Lyapunov criterion, we can verify that the stability of the system and the convergence of the third order sliding surface, i.e., $\sigma$, are guaranteed under the control law (47)-(51) despite whether the existing of the uncertainties or/and faults. Consequently, the stability and convergence of the second-order sliding surface, i.e., $\theta$, the first-order sliding surface, i.e., $s$, and the system state, i.e., $\chi$, are guaranteed according to the results in Theorems 1, 2 and 3. This completes the proof.

Remark 7. The proposed controller in (47)-(51) can be impleted as follows:

Step 1: Define the first-order sliding surface $\theta$, second-order sliding surface $s$ and third-order sliding surface $\chi$ as in (14), (15) and (16).

Step2: Calculate the equivalent controller in 48.

Step 3: Calculate adaptive fuzzy neural network controller (49) and its weight adaptation law 52.

290 Step 4: Calculate switching controller (50) using the adaptive law 51].

Step 5: Calculate the controller (47) and use it as the control input for the system. 
Remark 8. Even though the design of the integration of the FNN and the SMC is quite straightforward and this has been developed in many approaches in the literature, it is still novel to present it here since the design procedure is relatively different with the conventional integration techniques. For example, in this paper, according to (45) and (49), the integral FNN was used instead of the FNN.

Remark 9. In this paper, the effects of faults in the system are considered as the effects of an additional disturbance and the proposed PFTC based on TOFTSMC or adaptive fuzzy-neural TOFTSMC is designed as a robust controller to robust against the disturbances. Thus, the proposed TOFTSMC can be considered as an effective robust controller, which can be applied for wide applications to robust against the disturbance signals.

Remark 10. The selections of the adaptive gains $\lambda$ and $\alpha$ decide the convergent rate of the adaptive system. For example, if the parameters $\lambda$ and $\alpha$ are chosen as the high values, the convergent speed is faster, but it may lead to overestimated of the desired values. In practice, these values are usually chosen based on experiments.

\section{Results and discussions}

In this section, we employ the proposed FTC to the tracking control of attitude control of a spacecraft to show its effectiveness. The considered nonlinear dynamic model of a spacecraft used in this paper was taken as the same model as in Liang Y et al [3]. The dynamics model of the system can be described as the same form as (1) with $n=3$, in which the system parameters are $\chi=\left(\chi_{1}, \chi_{2}\right)^{T}, \chi_{1}=\left(x_{1}, x_{2}, x_{3}\right)=\left(\phi_{a}, \theta_{a}, \psi_{a}\right), \chi_{2}=$ $\left(x_{4}, x_{5}, x_{6}\right)=\left(\dot{\phi}_{a}, \dot{\theta}_{a}, \dot{\psi}_{a}\right), \mathrm{u}=\left(u_{1}, u_{2}, u_{3}, u_{4}\right)^{T}, f(\chi)=\left[f_{1}(\chi), f_{2}(\chi), f_{3}(\chi)\right]^{T}$ and $\varpi=$ $\left[\varpi_{1}, \varpi_{2}, \varpi_{3}\right]^{T}$. Here, $\phi_{a}, \theta_{a}, \psi_{a}$ and the smooth functions, $f(\chi)$ and $G(\chi)$ are defined as

315 in Liang Y et al[3]. The initial condition is set as $x(0)=[-0.7,-0.07,1.5,0.3,1.3,-0.2]^{T}$ and the disturbance is $\varpi=0.5[\sin (t), \cos (2 t), \sin (3 t)]^{T}$.

In this paper, two new controllers, i.e., TOFTSMC and adaptive fuzzy neural TOFTSMC (AFN-TOFTSMC), are proposed. First, we verify the superior property of the TOFTSMC approach. Then, we verify the stability and convergence of the adaptive fuzzy neural 
network law. In order to verify the performance of the proposed TOFTSMC, we compare it with the recent state-of-the-art SMCs based on the nonsingular fast terminal sliding mode control (NFTSMC) [53, 36], the FOSMC [34] and the PID-based SMC [21, 23] and the previous work PID-NFTSMC [37]. The comparison between the controllers are fair as they are built upon the theory of sliding mode control and have quite similar level of complexity. The parameters of the controllers are fairly selected as shown in Table 2 .

To simulate the fault's effects in the dynamics of spacecraft, a fault function $\Delta u=$ $\left[(20+5 \cos (t))(10 s), 0.85 u_{2}(20 s), 0,0\right]^{T}$ is supplied. It means we assume that there exists a bias fault $\Delta u_{1}=20+5 \cos (t)$ occurring in the first actuator at the time $t=10 \mathrm{~s}$, and a $85 \%$ partial loss fault occurring in the second actuator at the time $t=20 \mathrm{~s}$. The tracking performances of the system in both normal and fault operations are shown in Figs. 1.3. Particularly, Figs. 1 and 2 show the position and velocity tracking performances of the three Euler's angles, respectively, while Fig. 3 shows the efforts of the four control inputs. For the sake of comparison, the tracking errors and convergent times (from the starting position) of these controllers are also reported in Tables 3 and 4 . From the results shown in Figs. 1 and 2, we can see that the conventional NFTSMC provided quite good tracking accuracy when the system is in normal operation. However, when the faults occurred, the NFTSMC provided low robustness to tackle the effects of faults; when the faults occurred at $t=10 \mathrm{~s}$, the system became unstable immediately. The FOSMC provided better performance compared to the NFTSMC for tackling the effects of the faults, as shown in Figs. 1 and 2 . On the other hand, the design of the sliding surface based on the PID can increase the transient response against the fault's effects in the system. For example, at the time that the fault occurred at around the time $t=10 \mathrm{~s}$ and $t=20 \mathrm{~s}$, the PID-based SMC, the PID-NFTSMC and the proposed TOFTSMC have faster convergent speed than that of the NFTSMC and the FOSMC. For the faults occurred in the first actuator, the PID-NFTSMC provided higher performance than the PID-based SMC, as shown in Fig. 1. a) and Fig. 2(a). However, for the fault occurred in the second actuator, it provided big oscillatory, as shown in Fig. 11.b) and Fig. 2 b), which decreased the tracking performance of the system significantly.

This symptom might be caused by the effects of the differential term in the PID gains 
of the PID-NFTSMC. To eliminate this symptom of the PID-NFTSMC, an effective tuning mechanism for the PID gains may need to be investigated. On the other hand, because the TOFTSMC possesses the advantages of the FTSMC, the PID-based SMC and the HOSM simultaneously, it had a better performance compared to the PID-based SMC and the PID-NFTSMC, and much better performance compared to the NFTSMC and the FOSMC in both tracking precision and convergent time, as shown in Tables 3 and 4 Particularly, the proposed TOFTSMC provided very high tracking performance when the system is in normal operation, whilst when the faults occurred, it compensated the effects of faults very well, as shown in Figs. 1 and 2 In addition, as shown in Fig. 3, the TOFTSMC provided smooth control efforts compared to other controllers such as the NFTSMC, the FOSMC, the PID-based SMC and the PID-NFTSMC. Thus, we can conclude that, compared to the NFTSMC, the FOSMC, the PID-based SMC and the PID-NFTSMC, the proposed TOFTSMC provided the best performance for the system in terms of tracking precision, fast transient response, finite time convergence and chattering elimination. This verified the expected properties of the proposed controller compared to other advanced sliding mode controllers shown in Table 1 .

In the next, we verify the performance of the AFN-TOFTSMC to verify the effectiveness of the integration of FNN into the system. A set of membership functions of FNN used in this paper are constructed as

370 $\quad \mu_{A_{j}^{1}}=\exp \left(-\left(\chi_{i}+5\right)^{2} / 4\right), \mu_{A_{j}^{2}}=\exp \left(-\left(\chi_{i}+3\right)^{2} / 4\right), \mu_{A_{j}^{3}}=\exp \left(-\left(\chi_{i}+1\right)^{2} / 4\right), \mu_{A_{j}^{4}}=$ $\exp \left(-\left(\chi_{i}+0\right)^{2} / 4\right), \mu_{A_{j}^{5}}=\exp \left(-\left(\chi_{i}-1\right)^{2} / 4\right), \mu_{A_{j}^{6}}=\exp \left(-\left(\chi_{i}-3\right)^{2} / 4\right), \mu_{A_{j}^{7}}=\exp \left(-\left(\chi_{i}-5\right)^{2} / 4\right)$.

The position tracking performance of the AFN-TOFTSMC was shown in Fig. 4. Comparison results between Fig. 1 and Fig. 4 shown that the proposed AFN-TOFTSMC has the same important property as the TOFTSMC; the proposed AFN-TOFTSMC compensated the fault's effects very well without requiring the prior knowledge of the bounded value of the uncertainties and faults and the exact dynamics function. The time responses of the third-order sliding surface of the AFN-TOFTSMC was shown in Fig. 5. From Fig. 5, we can see that the proposed sliding surface converged to zero very quickly. In addition, from the results shown in Fig. 4 and Fig. 5 it can be seen that all three orders of the sliding surfaces proposed in $(8),(9)$ and $(10)$ were stable and convergent. The three outputs of the FNN were shown in Fig. 6 From Fig. 6 , we 


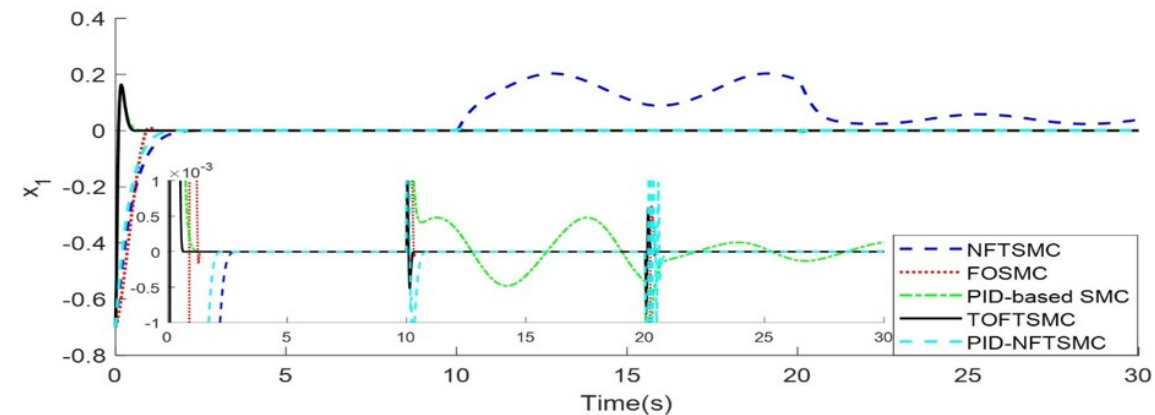

(a)
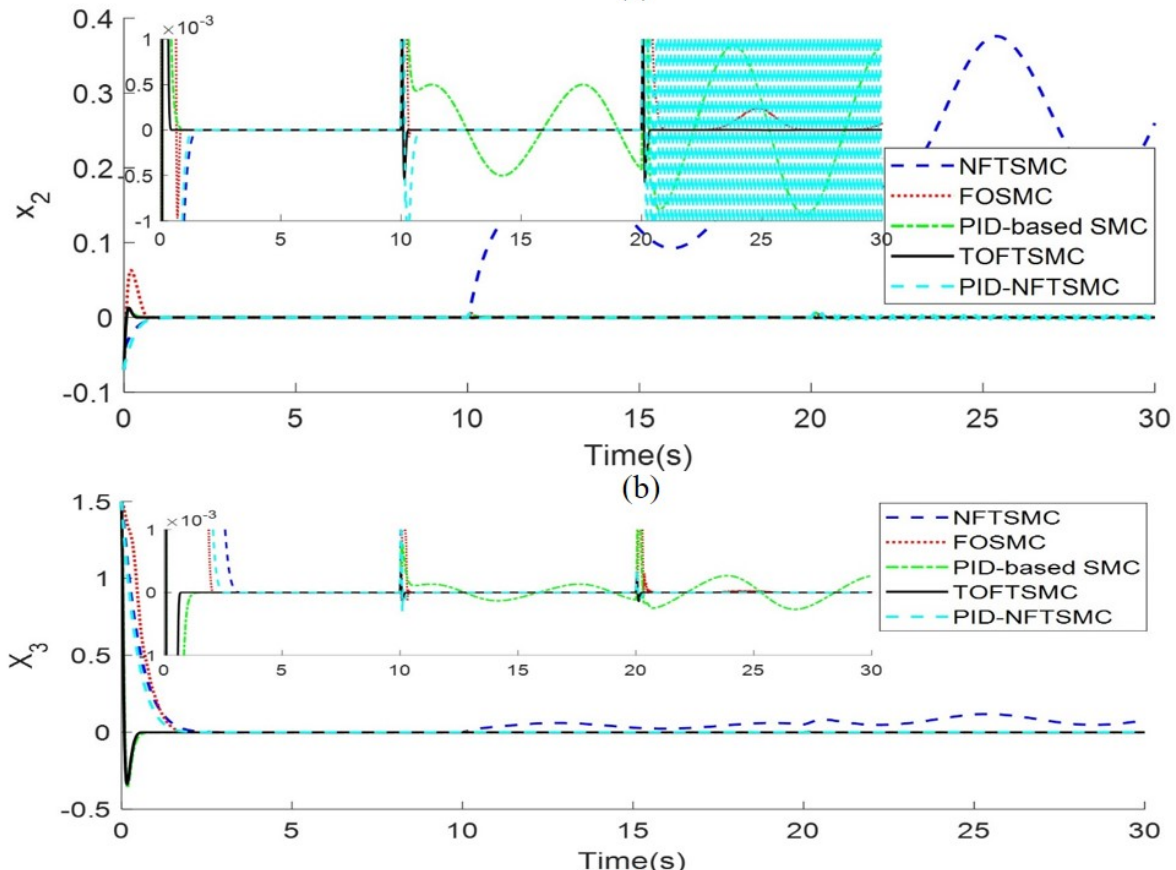

(c)

Figure (1) Time history of system states $x_{1}, x_{2}, x_{3}$ of the NFTSMC, the FOSMC, the PID-based SMC, the PID-NFTSMC and the TOFTSMC controllers 


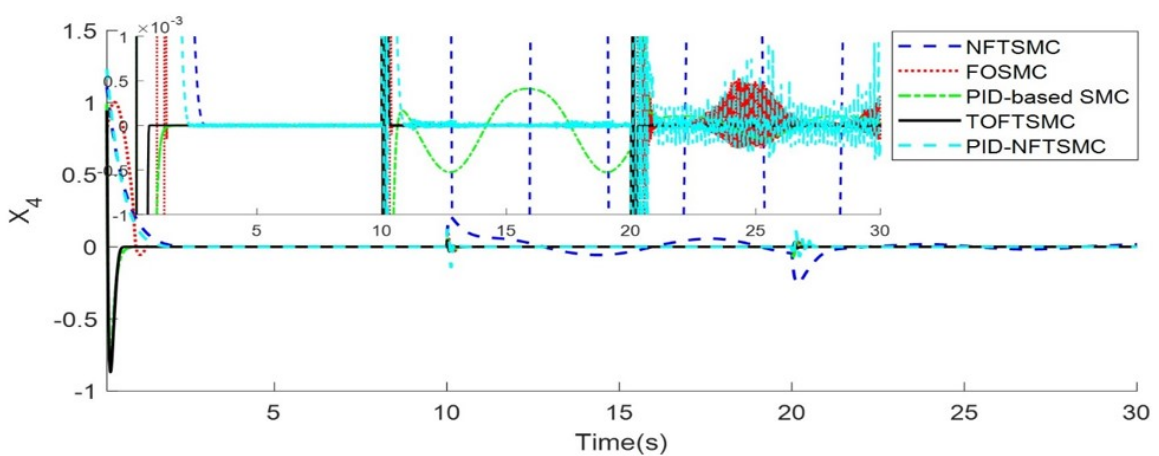

(a)
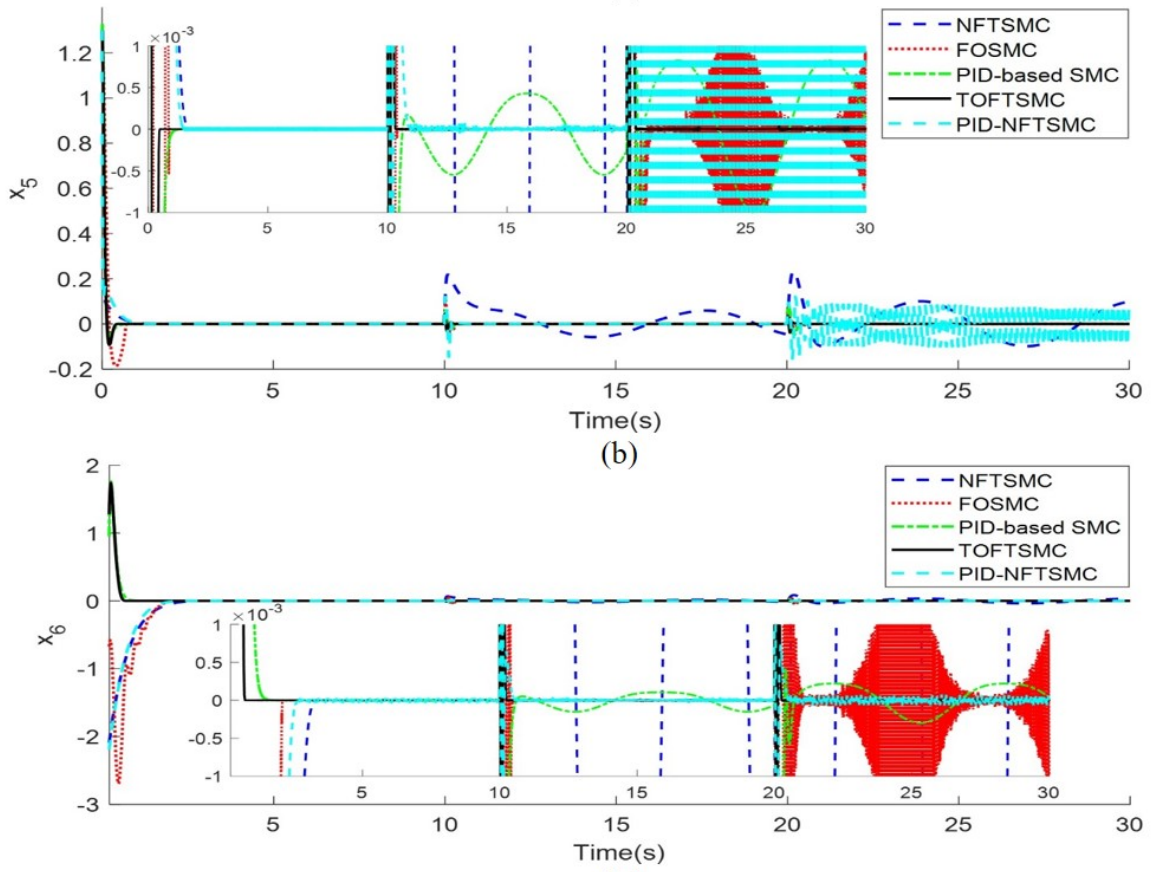

(c)

Figure (2) Time history of system states $x_{4}, x_{5}, x_{6}$ of the NFTSMC, the FOSMC, the PID-based SMC, the PID-NFTSMC and the TOFTSMC controllers 


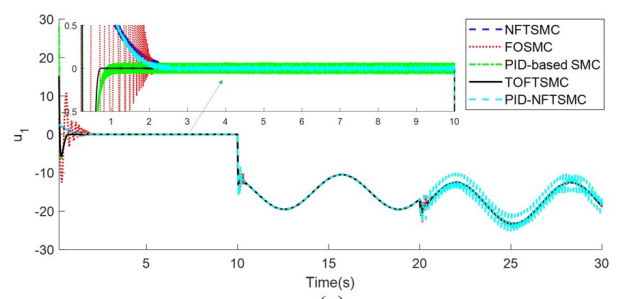

(a)

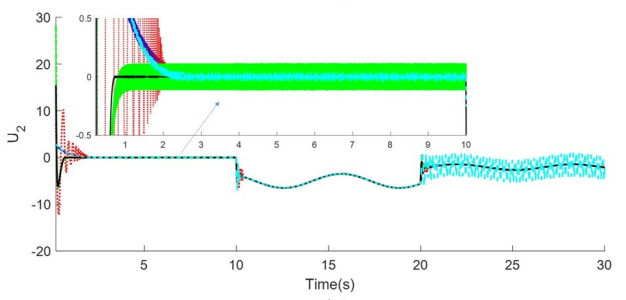

(b)
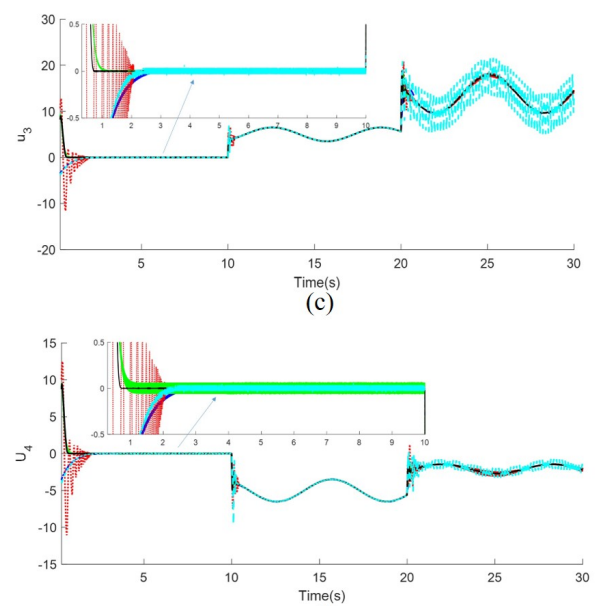

(d)

Figure (3) Time history of four control inputs of the NFTSMC, the FOSMC, the PID-based SMC, the PID-NFTSMC and the TOFTSMC controllers

can see that, when the system in normal operation $(t<10 s)$, the FNN approximated the uncertainty components, whilst, when the system in fault operation $(t>10 s)$, the FNN tent to approximate both the uncertainties and faults. The results are identical to the analysis as in the section 4. Therefore, we can conclude that the FNN system works well for the system. Fig. 7 shown the convergence of the adaptive gain $K_{\phi}$ of the proposed adaptive law (50) of the proposed AFN-TOFTSMC.

Remark 11. In this simulation, there are no specific physical meaning behind the uses of sin and cos functions in the assumed disturbance and faul components, i.e., $\varpi$ and $\Delta u_{1}$. The assumed disturbance and fault components are selected as arbitrary functions and they could be replaced by another functions.

Remark 12. Because the AFN-TOFTSMC has the similar property to the TOFTSMC, the velocity tracking performance $\left(x_{4}, x_{5}, x_{6}\right)$ and the control inputs of the AFN-TOFTSMC are omitted in this section for the purpose of reducing the length of the paper.

395 Remark 13. For the FNN system, the approximating precision depends on the number of membership functions used. If the number of membership functions are large, the approximation accuracy is higher, but the computational time is larger, and vice versa. 
This paper selected seven membership functions as mentioned in the simulation section to guarantee both good approximation and acceptable computational burden.

400 Remark 14. Based on the obtained results in the previous section, the pros and cons of the proposed method can be listed as follows. Pros: (i) provides fast transient response and finite convergence, (ii) provides high tracking precision, i.e., low steadystate-error, and (iii) provides almost smooth control efforts. Cons: the proposed sliding surface creates many tuning parameters that need to be effectively selected to get higher performance.

Remark 15. According to (50), the switching term, which contained sign function, is under integral, thus, the chattering in the system is much reduced but not totally eliminated. To further reduce the chattering, the switching term can be replaced by the following function.

$$
\dot{u}_{a n}=-\left(\hat{K}_{\phi}+\eta\right)\left(\frac{\sigma}{|\sigma|+\kappa}\right)
$$
sliding surface. From our experiments, this component is important for FTC system since it increases the robustness of the system so that the system can compensate the effects of faults quicker. As a result, the system could have more opportunities to avoid 


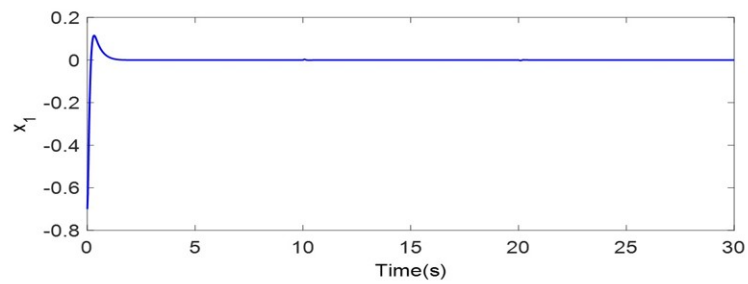

(a)

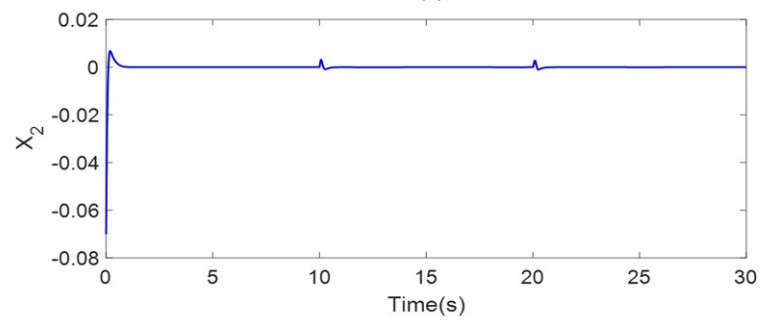

(b)

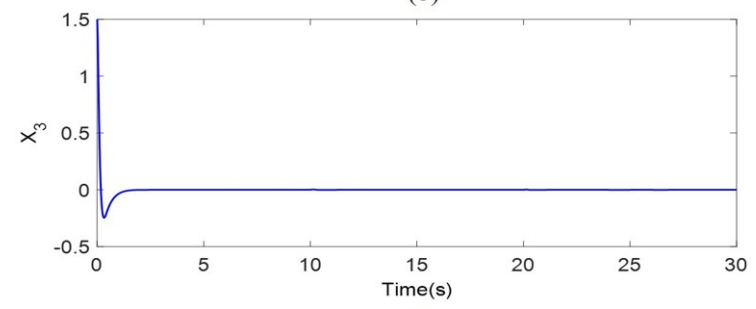

(c)

Figure (4) Time history of system states $x_{1}, x_{2}, x_{3}$ under the effect of fault $\Delta u$ and the AFN-TOFTSMC controller

'exploding'. When the system is converged, the system becomes continuous again (unless another faults occur) and the Lemmas 1 and 2 are now applicable. This is one of the most contributions of this paper. To support this statement, in the simulation results, the effects of abrupt fault were simulated instead of incipient faults to see the performance of the system. From the simulation results, it can be seen that the fault effects were compensated very quickly and the system almost became smooth.

\section{Conclusions}

A robust fault tolerant control based on a novel TOFTSMC was developed for a class of second-order nonlinear systems. By taking the merits of the individual approach based on the FTSMC, the PID-based SMC and the HOSM controllers into 

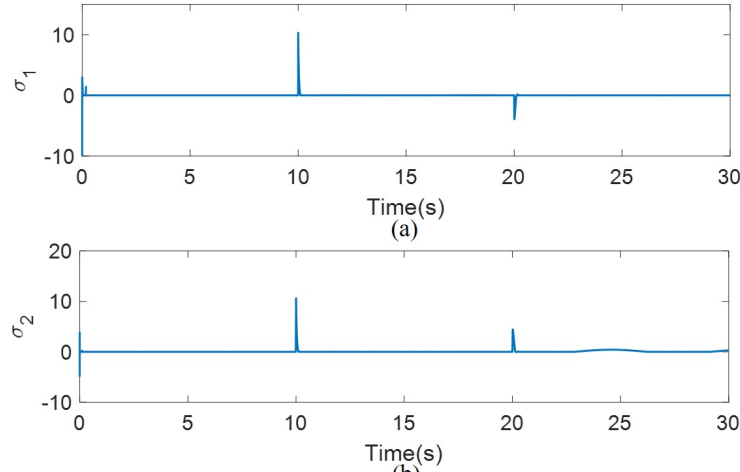

(b)

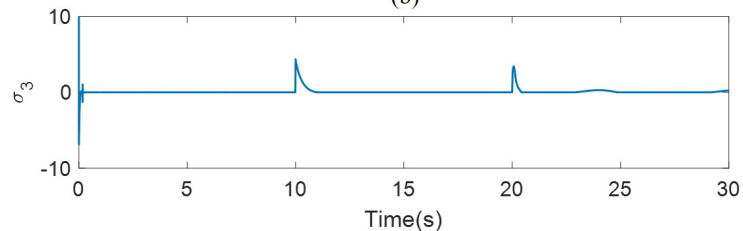

(c)

Figure (5) Time history of sliding surface $\sigma$ of the AFN-TOFTSMC controller

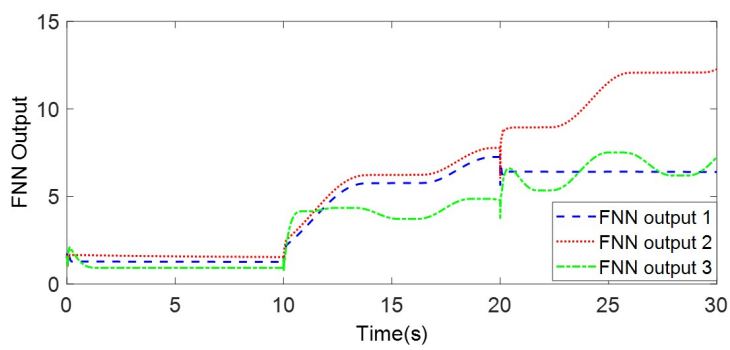

Figure (6) FNN's outputs of the AFN-TOFTSMC controller

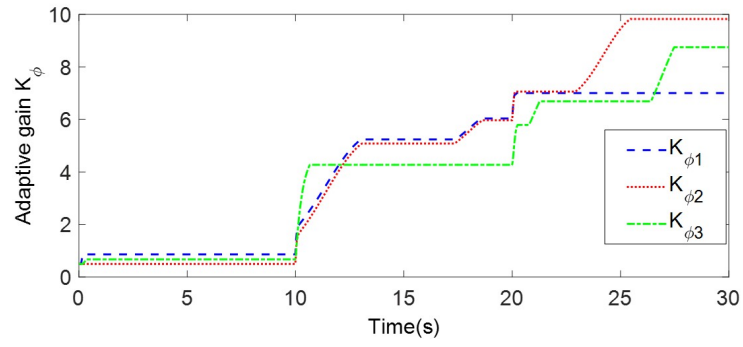

Figure (7) The variant of the adaptive gain $K_{\phi}$ of the AFN-TOFTSMC controller 
Table (2) Selected parameters of the controllers

\begin{tabular}{lll}
\hline \hline & & \\
Controller & Parameters & Value \\
\hline \multirow{2}{*}{ NFTSMC } & $\sigma_{1}, \sigma_{2}, \alpha, p, q, \rho_{d}, \psi$ & $\operatorname{diag}\{10,10,10\}, \operatorname{diag}\{5,5,5\}, 1.4,9,7,20,1000$ \\
FOSMC & $c_{1}, c 2, \alpha_{1}, \alpha_{2}, \Lambda, k_{d}+k_{T}, \eta$ & $10,7,9 / 23.9 / 16,0.5,20,0.5$ \\
PID-based SMC & $k, \lambda, \psi$ & $10,5,1000$ \\
PID-NFTSMC & $K_{p}, K_{i}, K_{d}$ & $5,100,0.02$ \\
& Other parameters & Same as the NFTSMC \\
TOFTSMC & $h_{1}, h_{2}, h_{3}, \gamma_{1}, \gamma_{2}$ & diag $\{10,10,10\}, \operatorname{diag}\{5,5,5\}, \operatorname{diag}\{5,5,5\}, 7 / 9,1.4$ \\
& $k_{1}, k_{2}, \varphi_{1}, \varphi_{2}$ & diag $\{10,10,10\}, \operatorname{diag}\{5,5,5\}, 7 / 9,1.4$ \\
& $m_{1}, m_{2}, \alpha_{1}, \alpha_{2}$ & diag $\{10,10,10\}, \operatorname{diag}\{5,5,5\}, 7 / 9,1.4$ \\
& $\Lambda, k_{T}, \eta$ & $0.5,20,0.5($ same as the FOSMC) \\
AFN-TOFTSMC & Adaptive gains $\lambda, \alpha$ & $1 / 3,1 / 4$ \\
& Other parameters & Same as the TOFTSMC controller \\
\hline \hline
\end{tabular}

Table (3) Comparison in root mean square position error and convergence time (CT) of position tracking error of the controllers when the fault occurs

\begin{tabular}{lllllll}
\hline \hline Controller & $x_{1}$ & $x_{2}$ & $x_{3}$ & $C T_{1}$ & $C T_{2}$ & $C T_{3}$ \\
\hline & & & & & & \\
NFTSMC & 0.1136 & 0.1766 & 0.1515 & 2.92 & 1.71 & 3.30 \\
FOSMC & 0.0702 & 0.0060 & 0.1264 & 1.36 & 0.83 & 2.01 \\
PID-based SMC & 0.0399 & 0.0026 & 0.0864 & 1.05 & 0.85 & 1.05 \\
PID-NFTSMC & 0.0570 & 0.0049 & 0.1291 & 1.98 & 1.25 & 2.35 \\
TOFTSMC & 0.0326 & 0.0020 & 0.0687 & 0.59 & 0.39 & 0.58 \\
\hline \hline
\end{tabular}


Table (4) Comparison in root mean square velocity error of the controllers when the fault occurs

\begin{tabular}{llll}
\hline \hline & & & \\
Controller & $x_{4}$ & $x_{5}$ & $x_{6}$ \\
\hline & & & \\
NFTSMC & 0.4013 & 0.0642 & 0.8636 \\
FOSMC & 0.3289 & 0.0665 & 0.7099 \\
PID-based SMC & 0.1394 & 0.0516 & 0.2698 \\
PID-NFTSMC & 0.1445 & 0.0429 & 0.2920 \\
TOFTSMC & 0.1372 & 0.0441 & 0.2694 \\
\hline \hline
\end{tabular}

account, the proposed TOFTSMC possesses many great properties such as strong robustness, less steady-state error, chattering-free and fast finite time convergence. In addition, in order to exclude the drawbacks of the TOFTSMC such as the system was designed based on the assumptions that the exact function dynamics and the prior knowledge of the bounded value of the uncertainties and faults must be known in advance, an adaptive fuzzy neural network was then designed; yielding a new adaptive fuzzy neural TOFTSMC (AFN-TOFTSMC). The proposed methods were then applied to attitude control of a spacecraft. The results shown that, when comparing to other stateof-the-art approaches such as the NFTSMC, the FOSMC, the PID-based SMC and the PID-NFTSMC, the proposed approaches, i.e.,TOFTSMC and AFN-TOFTSMC, provide much higher fault compensation capability.

The implementation procedure of the proposed fuzzy neural TOFTSM controller stated in Remark 7 is quite straightforward. Therefore, it can be easily implemented in the practical systems. Actually, the proposed approach provides many advantages for the practical systems: (i) it robusts against the effects of the uncertainties and faults, (ii) the chatteing is significantly reduced or eliminated, which is particularly useful when implementing into practical system to avoid mechanical oscillations, (iii) the proposed approach does not require the knowledge of dynamic model, which make it easier to be implemented into the practical systems. 


\section{Future recommendation}

In this paper, only the gain fault and bias fault of actuators were considered and solved. In addition, the constraints on the control inputs and outputs of the system, which are required for some practical applications, have not been considered yet. In the future work, we will investigate the performance of the proposed method for other types of actuator faults and sensor faults and investigate the performance of the proposed method for the constraints system. On the other hand, the finite controller designed depends on the initial conditions of the states. How to remove this condition will be studied in the future work.

\section{Conflict of interest}

The authors declare that they have no conflict of interest.

\section{References}

${ }_{465}$ [1] M. Van, S. S. Ge, D. Ceglarek, Fault estimation and accommodation for virtual sensor bias fault in image-based visual servoing using particle filter, IEEE Transactions on Industrial Informatics 14 (4) (2018) 1312-1322.

[2] M. Van, H. Kang, Bearing defect classification based on individual wavelet local fisher discriminant analysis with particle swarm optimization, IEEE Transactions on Industrial Informatics 12 (1) (2016) 124-135.

[3] Y. Liang, S. Xu, C. Tsai, Study of vsc reliable designs with application to spacecraft attitude stabilization, IEEE Transactions on Control Systems Technology 15 (2) (2007) 332-338.

[4] Z. Gao, C. Cecati, S. X. Ding, A survey of fault diagnosis and fault-tolerant techniques-part i: Fault diagnosis with model-based and signal-based approaches, IEEE Transactions on Industrial Electronics 62 (6) (2015) 3757-3767. 
口 [5] S. Tabatabaeipour, R. Izadi-Zamanabadi, T. Bak, A. Ravn, Passive fault-tolerant control of discrete time piecewise affine systems against actuator faults. International Journal of Systems Science 43 (11) (2012) 1985-1997. arXiv:https: //doi.org/10.1080/00207721.2012.670289 URL https://doi.org/10.1080/00207721.2012.670289

[6] A. Paoli, M. Sartini, S. Lafortune, Active fault tolerant control of discrete event systems using online diagnostics, Automatica 47 (4) (2011) 639 - 649.

[ URL http://www.sciencedirect.com/science/article/pii/ S0005109811000227

[7] M. Benosman, K. . Lum, Passive actuators' fault-tolerant control for affine nonlinear systems, IEEE Transactions on Control Systems Technology 18 (1) (2010) $152-163$.

[8] R. Wang, J. Wang, Passive actuator fault-tolerant control for a class of overactuated nonlinear systems and applications to electric vehicles, IEEE Transactions on Vehicular Technology 62 (3) (2013) 972-985.

[9] Y. Li, G. Yang, Model-based adaptive event-triggered control of strict-feedback nonlinear systems, IEEE Transactions on Neural Networks and Learning Systems 29 (4) (2018) 1033-1045.

[10] Y. Li, G. Yang, Observer-based fuzzy adaptive event-triggered control codesign for a class of uncertain nonlinear systems, IEEE Transactions on Fuzzy Systems 26 (3) (2018) 1589-1599.

[11] V. Utkin, Sliding Modes on control and Optimization, Springer Verlag, Germany, 1992.

[12] X.-T. Tran, H.-J. Kang, Robust adaptive chatter-free finite-time control method for chaos control and (anti-)synchronization of uncertain (hyper)chaotic systems, Nonlinear Dynamics 80 (1) (2015) 637-651.

URL https://doi.org/10.1007/s11071-015-1895-6 
[13] B. Zhao, B. Xian, Y. Zhang, X. Zhang, Nonlinear robust sliding mode

505

$10.1002 /$ rnc.3290

URL/https://onlinelibrary.wiley.com/doi/abs/10.1002/rnc.3290

510

[14] H. Imine, T. Madani, Sliding-mode control for automated lane guidance of heavy vehicle, International Journal of Robust and Nonlinear Control 23 (1) (2011) 6776. arXiv:https://onlinelibrary.wiley.com/doi/pdf/10.1002/rnc. 1818 .

URL https://onlinelibrary.wiley.com/doi/abs/10.1002/rnc.1818

[15] Y. Liang, S. Xu, D. Liaw, C. Chen, A study of t-s model-based smc scheme with application to robot control, IEEE Transactions on Industrial Electronics 55 (11) (2008) 3964-3971.

[16] B. Xiao, Q. Hu, Y. Zhang, Adaptive sliding mode fault tolerant attitude tracking control for flexible spacecraft under actuator saturation, IEEE Transactions on

[17] Q. Hu, B. Xiao, Fault-tolerant sliding mode attitude control for flexible spacecraft under loss of actuator effectiveness, Nonlinear Dynamics 64 (1) (2011) 13-23.

URL https://doi.org/10.1007/s11071-010-9842-z

[18] V. Parra-Vega, S. Arimoto, Y.-H. Liu, G. Hirzinger, P. Akella, Dynamic sliding pid control for tracking of robot manipulators: theory and experiments, IEEE Transactions on Robotics and Automation 19 (6) (2003) 967-976.

[19] Y. Li, Q. Xu, Adaptive sliding mode control with perturbation estimation and pid sliding surface for motion tracking of a piezo-driven micromanipulator, IEEE Transactions on Control Systems Technology 18 (4) (2010) 798-810.

530 [20] J. Y. Peng, X. B. Chen, Integrated pid-based sliding mode state estimation and 
control for piezoelectric actuators, IEEE/ASME Transactions on Mechatronics 19 (1) (2014) 88-99.

[21] Y. Cao, X. B. Chen, An output-tracking-based discrete pid-sliding mode control for mimo systems, IEEE/ASME Transactions on Mechatronics 19 (4) (2014) $1183-1194$.

[22] F. Lin, Y. Hung, K. Ruan, An intelligent second-order sliding-mode control for an electric power steering system using a wavelet fuzzy neural network, IEEE Transactions on Fuzzy Systems 22 (6) (2014) 1598-1611.

[23] M. Van, S. S. Ge, H. Ren, Robust fault-tolerant control for a class of secondorder nonlinear systems using an adaptive third-order sliding mode control, IEEE Transactions on Systems, Man, and Cybernetics: Systems 47 (2) (2017) 221-228.

[24] M. Zhihong, A. P. Paplinski, H. R. Wu, A robust mimo terminal sliding mode control scheme for rigid robotic manipulators, IEEE Transactions on Automatic Control 39 (12) (1994) 2464-2469.

[25] R. Ma, G. Zhang, O. Krause, Fast terminal sliding-mode finite-time tracking con-

1. trol with differential evolution optimization algorithm using integral chain differentiator in uncertain nonlinear systems, International Journal of Robust and

(1) Nonlinear Control 28 (2) 625-639. arXiv:https://onlinelibrary.wiley. com/doi/pdf/10.1002/rnc.3890. URL https://onlinelibrary.wiley.com/doi/abs/10.1002/rnc.3890

[26] F. Lin, Y. Hung, K. Ruan, An intelligent second-order sliding-mode control for an electric power steering system using a wavelet fuzzy neural network, IEEE Transactions on Fuzzy Systems 22 (6) (2014) 1598-1611.

[27] H. Sun, S. Li, C. Sun, Finite time integral sliding mode control of hypersonic vehicles, Nonlinear Dynamics 73 (1) (2013) 229-244.

URL https://doi.org/10.1007/s11071-013-0780-4 
[28] Y. Cao, X. B. Chen, Disturbance-observer-based sliding-mode control for a 3-dof nanopositioning stage, IEEE/ASME Transactions on Mechatronics 19 (3) (2014) 924-931.

[34] Y. Feng, F. Han, X. Yu, Chattering free full-order sliding-mode control, Automatica 50 (4) (2014) 1310 - 1314.

URL http://www.sciencedirect.com/science/article/pii/ S0005109814000375

[35] S. Tong, B. Huo, Y. Li, Observer-based adaptive decentralized fuzzy fault-tolerant 
control of nonlinear large-scale systems with actuator failures, IEEE Transactions on Fuzzy Systems 22 (1) (2014) 1-15.

[36] M. Van, S. S. Ge, H. Ren, Finite time fault tolerant control for robot manipulators using time delay estimation and continuous nonsingular fast terminal sliding mode control, IEEE Transactions on Cybernetics 47 (7) (2017) 1681-1693.

[37] M. Van, An enhanced robust fault tolerant control based on an adaptive fuzzy pidnonsingular fast terminal sliding mode control for uncertain nonlinear systems, IEEE/ASME Transactions on Mechatronics 23 (3) (2018) 1362-1371.

[38] M. Van, M. Mavrovouniotis, S. S. Ge, An adaptive backstepping nonsingular fast terminal sliding mode control for robust fault tolerant control of robot manipulators, IEEE Transactions on Systems, Man, and Cybernetics: Systems (2018) 1-11doi:10.1109/TSMC. 2017.2782246.

[39] H. Alwi, C. Edwards, Fault tolerant control using sliding modes with on-line control allocation, Automatica 44 (7) (2008) 1859 - 1866. URL http://www.sciencedirect.com/science/article/pii/ S0005109807004803

[40] Y. . Liang, S. . Xu, Reliable control of nonlinear systems via variable structure scheme, IEEE Transactions on Automatic Control 51 (10) (2006) 1721-1726.

[41] M. Maki, J. Jiang, K. Hagino, A stability guaranteed active fault-tolerant control system against actuator failures, International Journal of Robust and Nonlinear

1. Control 14 (12) 1061-1077. arXiv:https://onlinelibrary.wiley.com/ doi/pdf/10.1002/rnc.932

URL https://onlinelibrary.wiley.com/doi/abs/10.1002/rnc.932

[42] Q. Hu, X. Shao, L. Guo, Adaptive fault-tolerant attitude tracking control of spacecraft with prescribed performance, IEEE/ASME Transactions on Mechatronics 23 (1) (2018) 331-341.

${ }_{610}$ [43] Y. Sun, L. Yao, Robust fault diagnosis and fault-tolerant control for non-gaussian uncertain stochastic distribution control systems. International Journal of Robust 
and Nonlinear Control 27 (10) 1709-1725. arXiv: https://onlinelibrary . wiley.com/doi/pdf/10.1002/rnc.3632.

URL https://onlinelibrary.wiley.com/doi/abs/10.1002/rnc.3632

[49] S. Tong, B. Huo, Y. Li, Observer-based adaptive decentralized fuzzy fault-tolerant control of nonlinear large-scale systems with actuator failures, IEEE Transactions on Fuzzy Systems 22 (1) (2014) 1-15.

[50] A. F. Filippov, Differential equations with discontinuous right hand side, Dordrecht, The Netherlands: Kluwer, 1988. 
[51] S. Tong, H.-X. Li, Fuzzy adaptive sliding-mode control for mimo nonlinear systems, IEEE Transactions on Fuzzy Systems 11 (3) (2003) 354-360.

[52] S. Tong, T. Wang, Y. Li, Fuzzy adaptive actuator failure compensation control of uncertain stochastic nonlinear systems with unmodeled dynamics, IEEE Transactions on Fuzzy Systems 22 (3) (2014) 563-574.

${ }_{645}$ [53] S. S. Xu, C. Chen, Z. Wu, Study of nonsingular fast terminal sliding-mode faulttolerant control, IEEE Transactions on Industrial Electronics 62 (6) (2015) 39063913.

[54] B. Tian, Z. Zuo, X. Yan, H. Wang, A fixed-time output feedback control scheme for double integrator systems Automatica 80 (2017) 17 - 24.

[55] M. L. Corradini, A. Cristofaro, Nonsingular terminal sliding-mode control of nonlinear planar systems with global fixed-time stability guarantees, Automatica 95 (2018) $561-565$.

655 URL http://www.sciencedirect.com/science/article/pii/ S0005109818303248 